Observation

\section{BABYDIET, a feasibility study to prevent the appearance of islet autoantibodies in relatives of patients with Type 1 diabetes by delaying exposure to gluten}

To the Editor: The early introduction of foods containing gluten or cereal before the age of 3 months is associated with an increased risk of islet autoimmunity in childhood [1, 2]. A pilot study called BABYDIET is currently underway to determine whether primary intervention through delayed introduction of dietary gluten is feasible and could reduce the incidence of islet autoimmunity in high-risk first degree relatives of patients with Type 1 diabetes.

Newborn children are eligible if they are younger than 3 months and are offspring or siblings of patients with Type 1 diabetes and also have HLA genotypes that confer a high diabetes risk $(D R B 1 * 03-D Q A 1 * 0501-D Q B 1 * 0201 / D R B 1 * 04$ $D Q A 1 * 0301-D Q B 1 * 0302 ; D R B 1 * 04-D Q A 1 * 0301-D Q B 1 * 0302 /$ $D R B 1 * 04-D Q A 1 * 0301-D Q B 1 * 0302$ or DRB1*03-DQA1*0501$D Q B 1 * 0201 / D R B 1 * 03-D Q A 1 * 0501-D Q B 1 * 0201)$.

The parents of all children are informed of the potential benefits of gluten avoidance in animal models of autoimmune diabetes and of the increased risk of islet autoimmunity found in children who received diets containing gluten at an early age. Children of consenting parents are randomised to two dietary intervention groups, one that introduces gluten-containing cereals at 12 months (late exposure) and one that introduces gluten at 6 months of age in line with national feeding guidelines (control group). Each family is visited by a nutritionist who explains which cereals contain gluten, and what food should be avoided during the intervention period. Lists of the most common foods introduced during the first year of life are examined and foods and products containing gluten are indicated. Details of gluten-free commercial infant products available in Germany are provided to the parents. Children are monitored intensively with 3-monthly collection of venous blood, urine and stool, and 3-day dietary records of weighed food intake for 3 years and yearly thereafter. Infections, medication and the introduction of new food groups are recorded daily. Additional demographic and family history questionnaires are collected at 3,12, 24 and 36 months of age, with questionnaires assessing the psychological impact collected at 3, 18 and 36 months. The study was approved by the ethics committee of the Ludwig-Maximilian University, Munich, Germany (Ethikkommission der Medizinischen Fakultät der Ludwig-Maximilians Universität No. 329/00).

DOI 10.1007/s00125-004-1420-9

Received: 6 February 2004 / Accepted: 19 April 2004

Published online: 28 May 2004

(C) Springer-Verlag 2004
Here we report the recruitment and compliance data in the first 24 months of the study. A total of 538 infants from Germany participated in the genetic screening and $56(10.4 \%)$ had one of the high-risk HLA genotypes. Fifty families (89\%) agreed to participate in the study and were randomised to one of the dietary groups ( 25 children to the late gluten exposure and 25 children to the control group). The children were monitored for a median of 15 months (range: 0-36). Three families/children withdrew from the study, two at age 6 months and one at age 18 months, stating that blood-drawing was the reason for withdrawal. Although no reliable confirmatory biomarker for compliance with dietary intervention was available, food records indicated that gluten was introduced at a median of 12 months (interquartile range: 12-12 months) in the group randomised to late gluten exposure and at 6.3 months (interquartile range: 6-7 months) in the control exposure group.

A total of 242 follow-up visits, including sampling of blood, urine and stool were scheduled for participants up to the age of 24 months. During these visits, 212 blood samples $(88 \%), 185$ stool samples $(76 \%)$ and 162 urine samples $(67 \%)$ were collected. Compliance with blood drawing and collection of urine and stool samples was similar at all follow-up visits and did not decrease with increasing age (Table $1, p=0.2$, chi square test for trend). Demographic questionnaires were completed by $93 \%$ of the families and questionnaires on psychosocial impact by $65 \%$ of mothers and $41 \%$ of fathers. Of 174 expected 3-day dietary records $122(70 \%)$ were returned. Daily records on infections, medication and food introduction were returned by $65 \%$ of the families. Compliance with all parts of the study was similar in the late-exposure and control groups. Of the 50 children, 32 children have reached 12 months of age or older. Of these 32 children, 21 (66\%) have returned every requested blood sample, ten $(31 \%)$ every requested urine sample, $16(50 \%)$ every requested stool sample and 13 of $29(45 \%)$ every requested 3-day dietary record. Eight families were poorly compliant (including the three drop out families), accounting for $53 \%$ of all missing samples and $57 \%$ of all missing questionnaires.

The data show that dietary intervention in infants at high genetic risk of Type 1 diabetes is feasible. Intensive follow-up studies should include frequent blood sampling, which appears to be well accepted by the families, and should also provide incentives for compiling daily event records and 3-day dietary records of weighed food intake, these last two being the least well complied with components of the study.

Acknowledgements. This study was supported by grants from the German Research Foundation (Deutsche Forschungsgemeinschaft ZI 310/14-1 and 14-2) and the Foundation for Children with Type 1 Diabetes (Stiftung 'Das Zuckerkranke Kind'). We thank Ulrike Mollenhauer, Andrea Baumgarten, Karolina v. Dalwigk and Katharina Warncke for expert technical assistance. We also thank all participating families and German paediatricians for their participation in the study.

S. Schmid, D. Buuck, A. Knopff, E. Bonifacio, A. G. Ziegler Diabetes Research Institute, Munich, Germany 
Table 1. Number of samples, questionnaires and protocols completed by participating families

\begin{tabular}{|c|c|c|c|c|c|c|c|c|}
\hline & \multicolumn{8}{|c|}{ Age at follow-up visit } \\
\hline & 3 months & 6 months & 9 months & 12 months & 15 months & 18 months & 21 months & 24 months \\
\hline \multicolumn{9}{|l|}{ Completed visits } \\
\hline Blood & $45(96 \%)$ & $38(88 \%)$ & $34(89 \%)$ & $28(88 \%)$ & $25(83 \%)$ & $19(83 \%)$ & $15(94 \%)$ & $8(62 \%)$ \\
\hline $\begin{array}{l}\text { Demographic } \\
\text { questionnaires }\end{array}$ & $47(100 \%)$ & - & - & $29(91 \%)$ & - & - & - & $10(77 \%)$ \\
\hline $\begin{array}{l}\text { 3-day dietary } \\
\text { records }\end{array}$ & - & $25 / 40(63 \%)$ & $25 / 35(71 \%)$ & $21 / 29(72 \%)$ & $19 / 27(70 \%)$ & $17 / 20(85 \%)$ & $10 / 13(77 \%)$ & $5 / 10(50 \%)$ \\
\hline
\end{tabular}

a The 3-day dietary records were requested from 6 months of age and introduced after the first three patients had been included; the total number of requested protocols at each follow-up is indicated by the number after the slash

\section{References}

1. Ziegler AG, Schmid S, Huber D, Hummel M, Bonifacio E (2003) Early infant feeding and risk of developing Type 1 diabetes-associated autoantibodies. JAMA 290:1721-1728

2. Norris JM, Barriga K, Klingensmith G et al. (2003) Timing of initial cereal exposure in infancy and risk of islet autoimmunity. JAMA 290:1713-1720
A. G. Ziegler ( $)$

Diabetes Research Institute, Koelner Platz 1, 80804 Munich, Germany E-mail: anziegler@1rz.uni-muenchen.de Tel.: +49-89-30793114

Fax: +49-89-3081733 